\title{
Kinetic pathways for the collapse of semiflexible polymers
}

\author{
G. G. Pereira* $\quad$ D. R. M. Williams ${ }^{\dagger}$
}

(Received 27 July 2003)

\begin{abstract}
Semiflexible polymers differ from their fully flexible counterparts in that the chain has a significant stiffness. As a result in poor solvent conditions the morphology of the condensed structure is a toroidal (rather than spherical) globule. In this paper we discuss the kinetic pathways to this toroidal globule from an extended state. The two possible pathways are (i) direct wind up and (ii) a series of metastable heads. The former collapse is rapid while the later is very slow.
\end{abstract}

\section{Contents}

\section{Introduction}

*School of Aerospace, Mechanical \& Mechatronic Engineering, University of Sydney, NSW, Australia. mailto: ggp@aeromech.usyd.edu.au

${ }^{\dagger}$ Department of Applied Mathematics, Australian National University, Canberra, Australia

See http://anziamj.austms.org.au/V45/CTAC2003/Pere for this article, (c) Austral. Mathematical Soc. 2004. Published April 10, 2004. ISSN 1446-8735 


\section{Introduction}

Polymer or macromolecules are ubiquitous in society today. For example a naturally occurring polymer is DNA which is the building block for life, while the simplest synthetic polymer is polyethylene, $\left(-\mathrm{CH}_{2}-\mathrm{CH}_{2}\right)_{n}$ which forms the basic ingredient for many plastics. In this paper we focus on a certain class of polymers known as semiflexible polymers. All polymers have a certain amount of stiffness (or inflexibility). Generally we model polymers as fully flexible chains and disregard this small stiffness. However for many polymers (for example DNA, Kevlar or F-actin) the stiffness of the chain is significant. For example, if a polymer chain is made up of $N$ monomers of length $b$ and diameter $d$ then the arclength of the chain $L=N b$. (Consider each monomer link as a cylinder of length $b$ and diameter $d$.) For fully flexible chains the persistence length, $\kappa$ (or the length over which the chain can remain straight) is of a similar order as $b$, that is, $\kappa \approx b$. However, for semiflexible chains the persistence length is much greater than $b$, that is, $\kappa \gg b$.

The conformation of a polymer chain is one of the most fundamental problems in polymer science. In a good solvent, that is, a solvent where the monomers and polymer will readily mix, the polymer chain will be extended. However, as one decreases the solvent quality, the monomers and solvent will eventually not mix readily. Such a solvent is commonly referred to as a poor solvent. In such a solvent, any contact between monomers and solvent incurs a large energy penalty. We shall refer to the polymer chain in a poor solvent as a condensed globule. The shape of this condensed globule will be such that the polymer chain adopts a conformation which tries to minimise 
solvent-polymer contact.

For a fully flexible chain, the conformation is one which will minimize the surface area for a given volume. In three dimensions (3D) the optimal shape of the condensed globule is a sphere. The radius of this spherical globule is $R_{\text {sphere }} \propto N^{1 / 3}$, since all monomers in the chains are contained in the globule. For a semi-flexible chain, excessive bending would occur if the chain condensed to a spherical globule. As a result, the shape of the condensed globule turns out to be a toroid, where the polymer chain wraps in circular rings around a central axis. In fact there exists an optimal radius for such a toroid determined by balancing the enthalpic energy (polymer-solvent contact) and bending energy of the stiff chain. Doing this one shows the radius size is $R_{\text {toroid }} \propto\left(L \kappa^{2} / \gamma^{2}\right)^{1 / 5}$, where $\gamma$ is the surface tension between polymer and solvent [1]. As a consequence, there is an optimal number of loops for a given toroid. Such toroids have also been seen experimentally [2]. An important question given the initial state is an extended (rod-like) configuration and the final state is a toroid is how does this toroid form, or what is the kinetic pathway from rod to toroid state and is there more than one pathway?

\section{Molecular simulations}

To probe the pathways for collapse of a semiflexible polymer chain from a extended configuration to a toroid we could in principle carry out experiments on single chains. Although this would be the preferred method, it is difficult because of the complicated experimental set-up and also the need to focus on particular parts of an isolated chain.

A useful alternative is to carry out "pseudo-computer experiments". That is, we simulate a polymer chain on a computer using an appropriate numerical model. Such simulations are quite common, nowadays, and have the advantage of being relatively cheap and easy to set-up [3]. Of course the major drawback of such a method is that these models are comparatively simple 
and do not contain all the physics that a true experiment would. However, when we focus on the major (molecular) factors that cause collapse we should, in principle, obtain useful insights and solutions of the problem at hand.

The molecular simulation procedure we use is that of Brownian Dynamics Simulations (BDS) which is quite similar to Molecular Dynamics Simulations (MDS). However, in BDS all monomer and solvent particles are not simulated as in MDS. Since many of the solvent particles do not do much, other than vibrate in space, we only physically simulate the polymer chain. Collisions with solvent molecules are effectively incorporated into an additional interaction (Gaussian noise term) with magnitude proportional to $k_{B} T$ where $k_{B}$ is Boltzmann's constant and $T$ is the temperature. Hence, we bypass the computationally expensive task of simulating all molecules in the system (as one would do in MDS) and only simulate the important molecule - the semiflexible chain. Further details of this method may be found in any good molecular simulation text [3, e.g.]. Here we only give details, relevant to our particular system.

We adopt a coarse grained model of the polymer chain where many monomers are incorporated into an effective bead [4]. The number of beads in our chain is denoted by $N$. In our simulations we use $N$ between 50 and 500 , focusing primarily on 200 beads. We use an off-lattice model, where beads may be anywhere in the 3D space. Bead $i$ is connected to beads $i-1$ and $i+1$ by Hookean springs, with spring constant $k$ and optimal bead spacing $l$. These three beads also form an angle, $\theta_{i}$, which we denote the bond angle. Since we simulate a semiflexible chain that prefers to be straight, this angle would optimally be zero radians. These are the only bonded molecular interactions we use. There is also the non-bonded interaction, which is the cause of the collapse of the chain to the condensed globule. This is the familiar Lennard-Jones (LJ) potential, with an excluded volume hard-core repulsion term plus a long ranged attractive tail. The sum of these three terms make up the entire molecular interactions for the polymer chain. For our purposes, it seems these three interactions are sufficient to induce col- 
(i)

(iii)
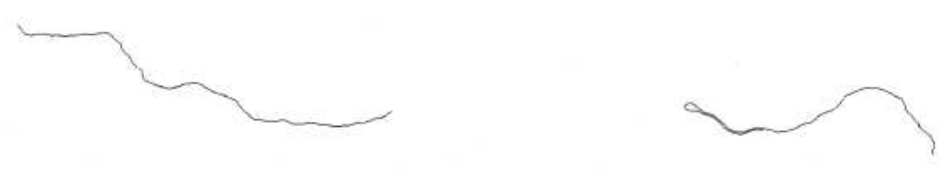

(iiii)

\section{(iv)}
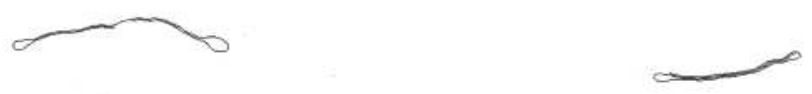

Figure 1: Snapshots (i) to (iv) for collapse via multiple headed tadpoles.

lapse. Thus it would seem this is the simplest model one could use to study semiflexible chain collapse. The total potential energy is therefore

$$
U=\frac{k}{2} \sum_{i=1}^{N}\left(\left|\mathbf{r}_{i}-\mathbf{r}_{i-1}\right|-l\right)^{2}+k_{B} T \frac{\kappa}{2 l} \sum_{i=1}^{N} \theta_{i}^{2}+\frac{\epsilon}{2} \sum_{\substack{i, j=1 \\ i \neq j}}^{N}\left(\frac{\sigma}{\left|\mathbf{r}_{i j}\right|}\right)^{12}-\left(\frac{\sigma}{\left|\mathbf{r}_{i j}\right|}\right)^{6},
$$

where $\mathbf{r}_{i}$ is the position of the $i$ th bead, $\epsilon$ is the magnitude of the LJ interaction between beads and $\sigma$ is an effective range of this interaction.

The dynamics of a bead is described by the Langevin equation

$$
m \ddot{\mathbf{r}}_{i}=-\nabla U_{i}-\zeta \dot{\mathbf{r}}_{i}-\eta_{i}(t),
$$

where $\zeta \dot{\mathbf{r}}_{i}$ is a drag force with frictional drag constant $\zeta$ and $\eta_{i}(t)$ is a Gaussian noise. The last two terms model the presence of the solvent. The friction and the the noise terms are related by the fluctuation dissipation theorem [3]:

$$
\left\langle\eta_{i}(t) \eta_{j}\left(t^{\prime}\right)\right\rangle=6 k_{B} T \zeta \delta_{i j} \delta\left(t-t^{\prime}\right) .
$$




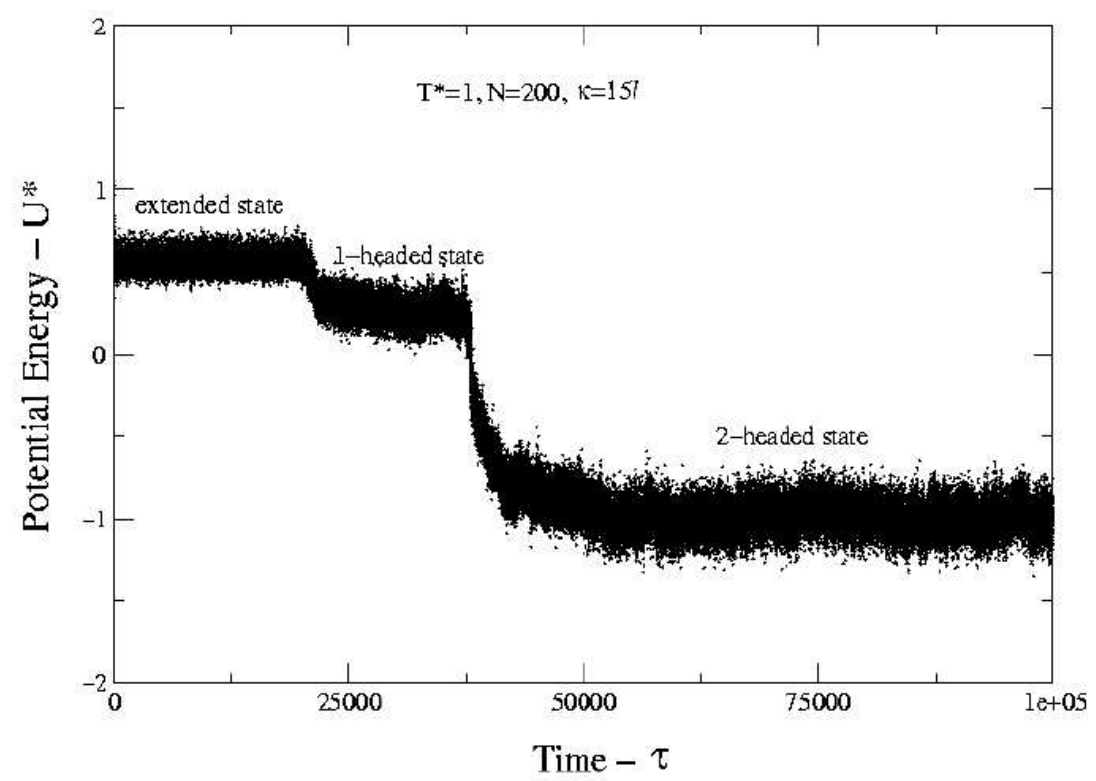

Figure 2: Potential energy versus time for metastable head collapse.

The Langevin equation (2) is a vectorial equation. We split it up appropriately into its 3 components. It is best to scale the Langevin equation appropriately. To do this we measure lengths with respect to $l$, that is, $\rho_{i}=r_{i} / l$, and energies with respect to $\epsilon$ so that the dimensionless potential energy is $U^{*}=U / \epsilon$, dimensionless temperature is $T^{*}=k_{B} T / \epsilon$ and time $\tau=\left(\epsilon / m l^{2}\right)^{1 / 2} t$. From now on all results will be given with respect to these dimensionless units. We use $\zeta=1$ which corresponds to moderate damping, and a velocity Verlet algorithm for integration with time steps of $\Delta \tau=10^{-4}$ to $10^{-2}$. 


\section{Results and discussion}

In our simulations we start the polymer chain in an extended (random) state and allow the system to run until the chain collapses. In all we ran approximately 100 simulations for chains with 200 beads. We use values $\kappa=15 l$, $k=200 k_{B} T / l^{2}$ and $T^{*}=1$. The final state of these runs were not necessarily always a toroid. It seems roughly $25 \%$ of our simulations ended up finally as toroids. Up to $75 \%$ of the simulations ended up in a multiple headed state. Figure 1 is an example of such a run. Initially, the semiflexible chain is an extended state. After moving around, due to thermal vibrations, one end of the chain will "touch" another part of the chain forming a closed loop. This event occurs due to random thermal vibrations, so in principle takes a long time. Once this occurs, the chain quickly "realizes" its potential energy may decrease if this end slides down the chain, as shown in the snapshot (ii). This state is referred to as a 1 headed tadpole. Due to the fact that the chain is comparatively long, it takes a while for the this end to make it completely to the other end. In the meantime the other end is also vibrating and in most cases will also form a closed loop. Subsequently, it also undergoes the sliding mechanism, so that the chain has 2 closed loops - a 2 headed tadpole (3rd snap-shot). In principle, this sliding procedure now proceeds so that the chain will become smaller and smaller (4th snap-shot). This process of sliding is quite slow and each of these $n$ headed states appear to be metastable states. In principle, the sliding proceeds so that the chain forms 3 heads, 4 heads and so on, until the toroid is reached with the optimal number of loops. In Figure 2 we show the corresponding potential energy as collapse occurs via a series of metastable heads. See in this figure that at each new head the potential energy has a discrete jump decrease which is the driving force for the collapse of the chain. Our simulations would have to proceed for inordinately long period of time for us to see the final toroidal state and so we have stopped at 2 heads.

There exists a second pathway to a collapsed toroidal globule. This process we refer to as direct wind up. In this process the chain initially vibrates 
(i)

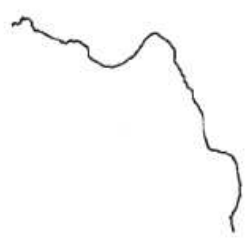

(iii)

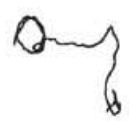

(ii)

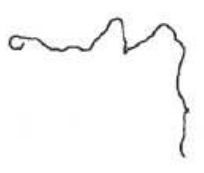

(iv)

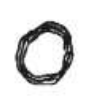

Figure 3: Snapshots (i) to (iv) for collapse via direct winding.

in the extended state and just as previously one end will eventually make a loop and contact another part of the chain. However, this contact is such that the chain continues to wind round the newly made loop. That is, the collapse proceeds via a wrapping mechanism rather than a sliding mechanism. Figure 3 shows the snap-shots where the first loop initially occurs and the last snapshot of the final toroid state. This collapse occurs very rapidly as shown in Figure 4. The actual collapse from the extended state to the toroidal state occurs in a single rapid interval.

We have also tried simulations on much longer chains of up to 500 beads. However, because of the much greater length of the chain the dynamics is much slower. When the chain increases the following qualitative observations are made:

1. initial collapse proceeds, generally at either end of the chain;

2. because of the much greater length of the chain, it seems both ends form 


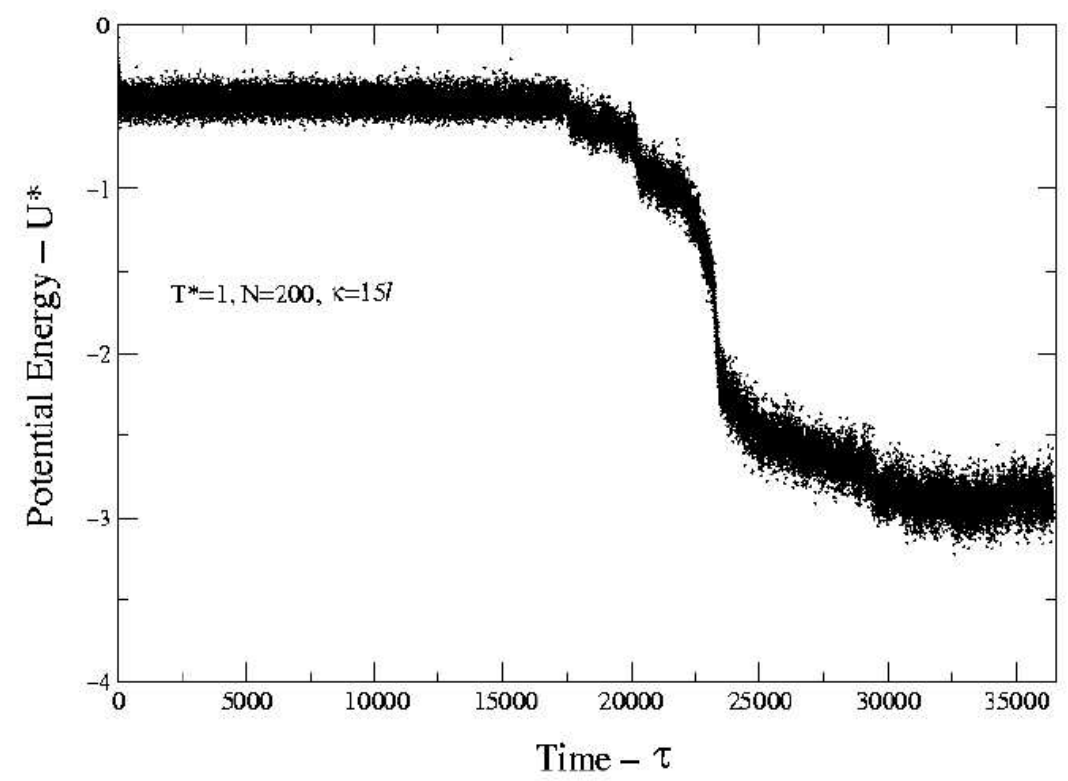

Figure 4: Potential energy versus time for direct wind-up collapse. 
loops so that collapse seems to be via a series of metastable heads;

3. however, there is an additional possibility of defects forming in the toroids where hairpins (or sharp, tight bends) also occur (this was also observed for 200 bead chains, but this event occurs much more often for longer chains).

In summary, we studied the kinetic pathways to toroidal collapse of a semiflexible polymer by Brownian Dynamical Simulations. We found collapse occurs via one of two possible pathways. The first pathway is via a series of metastable heads, and occurs predominantly. It is a slow collapse and involves the formation of 1 headed tadpole, 2 headed tadpole and so on until a toroid is formed. The primary mechanism in this pathway seems to be sliding of the chain. The second pathway is a direct wind up and occurs much less, but seems to be much faster. Here the primary mechanism is wrapping of the chain.

Acknowledgements: We acknowledge the help of Vislab computers and staff at the University of Sydney. GGP acknowledges the support of a QEII Fellowship.

\section{References}

[1] A. Yu. Grosbery and A. R. Khokhlov, Statistical physics of macromolecules, AIP Press, New York, 1994. C165

[2] V. A. Bloomfield, Biopolymers, 44, 269, (1998); V. V. Vasilevskaya, A. R. Khokhlov, S. Kidoaki and K. Yoshikawa, Biopolymers 41, 51, (1997). C165 
[3] M. P. Allen and D. J. Tildesley, Computer simulation of liquids, Clarendon Press, Oxford, 1987. C165, C166, C167

[4] K. Binder, Adv. Poly. Sci., 112, 181 (1994). C166 